\title{
EVALUACIÓN DEL DESARROLLO DE POLÍTICAS DE SEGURIDAD DE INFORMACIÓN
}

\author{
EVALUATION OF THE DEVELOPMENT OF THE INFORMATION \\ SECURITY POLICIES
}

\author{
${ }^{1}$ Richard Oswaldo Ticse Capcha; ${ }^{2}$ Henry George Maquera Quispe; ${ }^{3}$ Carlos Meza Quintana
}

\begin{abstract}
RESUMEN
En la actualidad la información de una institución se ha reconocido como un activo valioso y a medida que los sistemas de información apoyan cada vez más en los procesos generales, las tecnologias de información enfrentan amenazas de seguridad que incluyen, entre muchas otras: el fraude por computadora, espionaje, sabotaje, fuego, robo e inundación. Las posibilidades de daño y pérdida de información se hacen cada vez más comunes. El presente trabajo de Investigación permitió un diagnóstico sobre las políticas de seguridad en la Universidad Nacional del Centro del Perú, mediante la aplicación de técnicas metodológicas de auditoria y la evaluación de sus activos informáticos, para que la organización funcione correctamente y alcance los objetivos propuestos por su dirección. La meta de obtener un nivel considerable de seguridad se logrará con la propuesta que ofrece esta investigación mediante la "Implementación de Políticas de Seguridad de Información" para su aplicación. Esta investigación determina el diagnóstico de las políticas de seguridad de información comparando un antes y un después con y sin la implementación de políticas de seguridad; se obtuvo como resultado que las políticas de seguridad de información permiten una mejor gestión responsable de los activos de la información y además contar con estrategias de alto nivel para el control y administración efectiva de las mismas. El trabajo tuvo como propósito evaluar la situación actual en materia de seguridad informática, así como proponer una alternativa de solución basada en políticas de seguridad informática que fijen los mecanismos y procedimientos que se debe adoptar para salvaguardar sus sistemas y la información que estos contienen. Éstas políticas se diseñaron "a medida" para recoger las características propias como organización.
\end{abstract}

Palabras clave: Incidentes, amenaza, riesgo, vulnerabilidad, Seguridad informática, políticas de seguridad.

\begin{abstract}
At the moment, the information of an institution has been recognized as valuable assets and as the Information Systems support more and more in the general processes, Information Technologies face security threats that include, among others: computer fraud, spying, sabotage, fire, robbery and flooding. The possibilities of damage and information loss are more and more common. The present research allowed a diagnosis about the Security Policy at the National University of the Center of Peru, by means of the application of Audit Methodological techniques and the evaluation of its assets, so the organization works well and it reaches the proposed objectives for its management. The goal of getting an appreciable security level will be reached by using the proposal that suggest this research by the "Implementation of the Information Security Policy" for its application. This research determines the diagnosis of the Information Security Policy by making a before and after comparison with and without the implementation of security policies; as a result the Information Security Policies allows a better responsible management of the assets of information and although to rely on high-level strategies for the control and effective management of them all. The research had as a purpose the evaluation of the current situation in matters of Computer Science security, just like a an alternative solution based on Security policies that set all the procedures and mechanisms to adopt in order to safeguard the systems and information contained. These policies were designed "measured" to gather the own characteristics as an organization.
\end{abstract}

Key words: Incidents, threats, risk, vulnerability, security policies.

\section{INTRODUCCIÓN}

La Seguridad Informática está orientada a brindar protección contra las contingencias y riesgos relacionados con la infraestructura informática actualmente instalada en el Campus de la Universidad Nacional del Centro del Perú. La implementación de nuevas tecnologías y la adquisición e instalación de equipamiento informático requiere de un marco normativo que aporte las medidas inherentes a la seguridad; en el cual se plasmen mecanismos que resguarden su estado físico, su accesibilidad, uso y aprovechamiento; por esta razón, el objetivo del presente trabajo es estructurar Políticas de Seguridad Informática, con la finalidad de establecer un medio documental de consulta así como normativo para implementar las acciones que aporten medidas de seguridad y conservar en óptimo estado los bienes y servicios actualmente disponibles. 


\section{MATERIALES Y MÉTODOS}

El tipo de investigación que se ha llevado a cabo es descriptivo ya que se determina el diagnóstico de las políticas de seguridad de información en la Universidad Nacional del Centro del Perú. Para el desarrollo de la investigación se utilizó el marco de trabajo COBIT 4.1 bajo el siguiente esquema:

Objetivo PO9 - evaluar y gestionar los riesgos de TI. PO9 hace referencia a la Evaluación de Riesgos.

Planeación y Organización

- PO1 Definir un plan estratégico de tecnología de la información

- PO4Definir la organización y las relaciones de las TI

- PO5 Administrar la inversión en TI

Adquisición e Implementación

- AI2 Adquisición y mantenimiento de aplicaciones software

- AI3 Adquisición y mantenimiento de la infraestructura de la tecnología

Prestacióny Soporte

- DS4 Asegurar el servicio continuo

- DS5 Garantizar la seguridad del sistema

- DS11 Administrar los datos

- DS12 Administrar las instalaciones

Monitoreo

- M2 Evaluar lo adecuado del control interno.

También se realizaron entrevistas y encuestas al personal involucrado tendiendo como base normas o procesos de seguridad física, seguridad lógica, de comunicaciones, de aplicaciones, etc.

El método de estudio es el analítico puesto que evalúa de forma independiente cada componente de la seguridad de información para luego integrarlos como conjunto de solución.

\section{RESULTADOS}

Se identificó los principales activos y/o recursos más importantes a proteger con un nivel de importancia a criterio del Jefe de la Oficina General de Informática tal como se muestra en la Tabla $\mathrm{N}^{\circ} 1$. Cabe señalar también que para esta identificación se tomaron en cuenta los artículos 9 , 10,11 del Manual de Políticas de Seguridad Informática.

Tabla $N^{\circ} 1$. Activos/recursos a proteger y nivel de importancia

\begin{tabular}{|c|c|}
\hline $\mathrm{N}^{\circ}$ Recurso & Import. \\
\hline 1 Servidores & 10 \\
\hline 2 Data Center & 10 \\
\hline 3 Bases de Datos & 8 \\
\hline 4 Software de aplicaciones, programas fuente & 6 \\
\hline 5 BackUps & 5 \\
\hline $\begin{array}{l}\text { Información de configuraciones, datos en } \\
\text { medios externos }\end{array}$ & 10 \\
\hline $\begin{array}{l}7 \text { Cableado red y telefónico (Switchs, firewalls, } \\
\text { modems, antenas, etc.) }\end{array}$ & 7 \\
\hline $\begin{array}{l}8 \text { Hardware ( Pc's, portátiles, impresoras, } \\
\text { unidades de disco, etc.) }\end{array}$ & 5 \\
\hline $\begin{array}{l}9 \text { Equipos de Protección de energía (UPS, } \\
\text { baterías, etc.) }\end{array}$ & 2 \\
\hline 10 Personal Informático & 4 \\
\hline $\begin{array}{l}11 \text { Documentación de programas, hardware, } \\
\text { sistemas, manuales, etc. }\end{array}$ & 3 \\
\hline $\begin{array}{l}12 \text { Insumos (cartuchos de tinta, tóner, papel, } \\
\text { etc.) }\end{array}$ & 1 \\
\hline $\begin{array}{l}13 \text { Usuarios (Estudiantes, docentes, } \\
\text { administrativos, etc.) }\end{array}$ & 4 \\
\hline
\end{tabular}

De acuerdo a la diferencial hallada se puede elaborar los siguientes cuadrantes en la tabla $\mathrm{N}^{\circ} 2$ con los respectivos rangos de valores.

Tabla $\mathbf{N}^{\circ} 2$. Cuadrantes de riesgo

\begin{tabular}{cc}
\hline Cuadrante & Límites \\
\hline Riesgo Bajo & {$[133-811]$} \\
Riesgo Medio & {$[812-1490]$} \\
Riesgo Alto & {$[1491-2169]$} \\
\hline
\end{tabular}

Tabla $\mathbf{N}^{\circ}$ 3. Matriz categorizada

\begin{tabular}{|c|c|c|c|}
\hline $\mathbf{N}^{\circ}$ & Recurso & Vul. & Nivel \\
\hline 1 & Servidores & 2000 & Alto \\
\hline 2 & Data Center & 2167 & Alto \\
\hline 3 & Bases de Datos & 1633 & Alto \\
\hline 4 & Software de aplicaciones, programas fuente & 1275 & Medio \\
\hline 5 & Backups & 792 & Bajo \\
\hline 6 & Información de configuraciones, datos en medios externos & 1833 & Alto \\
\hline 7 & Cableado red y telefónico (Swtichs, firewalls, modems, antenas, etc.) & 1269 & Medio \\
\hline 8 & Hardware ( Pc's, portátiles, impresoras, unidades de disco, etc.) & 833 & Medio \\
\hline 9 & Equipos de Protección de energía (UPS, baterías, etc.) & 1050 & Medio \\
\hline 10 & Personal Informático & 842 & Medio \\
\hline 11 & Documentación de programas, hardware, sistemas, manuales, etc. & 600 & Bajo \\
\hline 12 & Insumos (cartuchos de tinta, tóner, papel, etc.) & 133 & Bajo \\
\hline 13 & Datos de usuarios (Estudiantes, docentes, administrativos, etc.) & 833 & Medio \\
\hline
\end{tabular}


Tabla $N^{\circ} 4$. Cuadro de indicadores COBIT 4.1

\begin{tabular}{|c|c|c|}
\hline Dominio & Indicador 1 & Indicador 2 \\
\hline \multirow{2}{*}{$\begin{array}{l}\text { Planeación y } \\
\text { Organización }\end{array}$} & Antigüedad del plan estratégico & $\%$ de áreas de negocio de usan TI \\
\hline & $\begin{array}{l}\text { Cantidad de soluciones tecnológicas que no están } \\
\text { alineadas con la estrategia de negocios }\end{array}$ & $\begin{array}{l}\% \text { de presupuesto de TI asignado a la } \\
\text { infraestructura de tecnologia }\end{array}$ \\
\hline \multirow[t]{2}{*}{$\begin{array}{l}\text { Adquisición e } \\
\text { Implementación }\end{array}$} & $\begin{array}{l}\text { Cantidad de aplicaciones entregadas puntualmente } \\
\text { de acuerdo al plan }\end{array}$ & $\begin{array}{l}\text { eantidad de pedidos de cambios relacionados con } \\
\text { defecto del sistema }\end{array}$ \\
\hline & $\begin{array}{l}\text { Cantidad de tecnologías y plataformas no } \\
\text { compatibles }\end{array}$ & \\
\hline \multirow[t]{3}{*}{ Entrega y Soporte } & $\%$ de contratos de servicios actualizados & \\
\hline & $\begin{array}{l}\text { \% utilización del personal TI en proceso de TI } \\
\text { que producen beneficios directos al negocios }\end{array}$ & $\begin{array}{l}\% \text { de proyectos que se realizaron con un } \\
\text { presupuesto de inversión de TI previamente }\end{array}$ \\
\hline & $\%$ de errores de entrada de datos & $\%$ de actualizaciones reprocesadas \\
\hline Monitoreo & $\begin{array}{l}\text { Cantidad, frecuencia y cobertura de informe de } \\
\text { cumplimiento interno }\end{array}$ & $\begin{array}{l}\text { Cantidad de acciones oportunas tomadas con } \\
\text { respecto a problemas de control interno }\end{array}$ \\
\hline
\end{tabular}

La tabla $\mathrm{N}^{\circ} 3$ muestra los activos/recursos a proteger con la descripción de los cuadrantes de acuerdo a los límites establecidos en la tabla $\mathrm{N}^{\circ} 2$.

La elaboración de indicadores tiene como base cada uno de los objetivos buscados, para poder cuantificar y/o cualificar y ver de esta manera si se está desarrollando de manera adecuada los procesos.

\section{DISCUSIÓN}

En la tabla $\mathrm{N}^{\circ} 5$ se muestra los activos o recursos ordenados según el nivel de riesgo en forma descriptiva. Por lo que podemos concluir que los recursos de TI en la UNCP se encuentran en un nivel de riesgo promedio; esto induce que actualmente no se está dando la debida importancia en temas de seguridad de las TI dentro de la institución, por lo que se ha elaborado e implementado Políticas de Seguridad Informática "a medida" de la Institución para su aplicación en los recursos con un nivel de riesgo alto y medio principalmente.

\section{CONCLUSIONES}

Con la implementación de Políticas de Seguridad
Informática en la UNCP actualmente se ha logrado una mejor gestión de TI, reduciendo los riesgos a los que se enfrenta los activos y/o recursos de TI.

La falta de inversión en recursos de TI en la Oficina General de Informática para implantar medidas de seguridad, provoca que la entidad tenga una exposición mayor a los riesgos en TI.

La Oficina General de Informática no cuenta con una definición bien establecida y aprobada de funciones y responsabilidades, en términos de seguridad, para la administración de los recursos informáticos respecto a las nuevas áreas que se van originando según las necesidades por las que atraviesa la UNCP.

A la fecha, la Oficina General de Informática está aplicando la seguridad a los activos más críticos de acuerdo a las necesidades según se estén presentando y a la disponibilidad de los recursos que permiten minimizar los riesgos.

No existe una evaluación bien establecida de los riesgos de las TI que permitan definir qué activos están más propensos frente a las amenazas que actualmente se encuentran.

Tampoco existe personal especializado operativo en temas de seguridad de TI en la Oficina que permita llevar

Tabla $N^{\circ} 5$. Matriz categorizada ordenada

\begin{tabular}{lll}
\hline & Recurso & Nivel \\
\hline 1 & Servidores (Aplicaciones, Base de Datos, Web y correo) & Alto \\
2 & Data Center & Alto \\
3 & Bases de Datos & Alto \\
6 & Información de configuraciones, datos en medios externos & Alto \\
4 & Software de aplicaciones, programas fuente (sistema académico, caja, personal, investigación, trámite & Medio \\
7 & Cableado red y telefónico (Swtichs, firewalls, modems, anterłas, etc.) & Medio \\
8 & Hardware (Pc's, portátiles, impresoras, unidades de disco, etc.) & Medio \\
9 & Equipos de Protección de energía (UPS, baterías, etc.) $\quad \mathbf{N}^{\circ}$ & Medio \\
10 & Personal Informático & Medio \\
13 & Datos de usuarios físicos (Estudiantes, docentes, administrativos, etc.) & Medio \\
5 & Backups & Bajo \\
11 & Documentación de programas, de hardware, sistemas, manuales, etc. & Bajo \\
12 & Insumos (cartuchos de tinta, tóner, papel, etc.) & Bajo \\
\hline
\end{tabular}


a cabo de manera correcta una mejor administración de los recursos informáticos.

\section{REFERENCIAS BIBLIOGRÁFICAS}

Bernal Montañez, R. (1994). Auditoria de los Sistemas de Información. España: UPV.

Borghello Cristian Fabián. (2001). Seguridad Informática. (Tesis). Argentina: Universidad Tecnológica Nacional.

Bravo González, A. L. (1998). La Información y la Auditoria de Sistemas. México: Universidad Iberoamericana.

Córdova Rodríguez Norma. (2003). Plan de Seguridad Informática para una Entidad Financiera. (Tesis). Perú: UNMSM.

Chapin, D. \& Akridge, S. (2005) ¿Cómo puede medirse la seguridad? Revisado en:

http://www.iso27000.es/download/HowCanSecur ityBeMeasured-SP.pdf.
Dolores Cerini M. y Ignacio Prá P. (2003). Plan de Seguridad en Informática. (Tesis). Argentina: Universidad Católica de Córdova.

Echenique García, J. A. (1997). Auditoria en Informática. Ed. México: McGraw-Hill.

Etcheverry, S. (2003). COBIT. Revisado en: http://www.unap.cl/ setcheve/cobit/

Fine H., L. (1998). Seguridad en centros de cómputo. Ed. México: Trillas.

ISACA (2007) COBIT 4.1. EE.UU.: IT Governance Institute.

Miro, I. (2005). Seguridad de la Información. Revisado en: http://www.kelssiler.com/

Oficina General de Planificación. (2010). Reglamento de Organización y Funciones. UNCP.

Oficina General de Planificación. (2010). Manual de Organización y Funciones. UNCP.

Pallavicini, C. (2005). Seguridad Informática. Revisado en: http://www.seguridadinfomatica.cl/empresa.php
Correspondencia:

Richard Oswaldo Ticse Capcha: rticsec@uncp.edu.pe

Henry George Maquera Quispe: henry.maquera@gmail.com
Fecha de Recepción: 06/10/2014

Fecha de Aceptación: 28/11/2014 


\section{NORMAS EDITORIALES Y GUÍA DE PRESENTACIÓN DE ARTÍCULOS CIENTÍFICOS PARA LA REVISTA CIENCIA \& DESARROLLO}

La Revista Cientifica Ciencia \& Desarrollo pone a consideración de la comunidad científica y académica, sus normas editoriales y guia de presentación de los artículos científicos, con la finalidad de lograr que la publicación de la revista sea un espacio abierto para la divulgación de nuevos conocimientos.

\section{CRITERIOS DE PUBLICACIÓN, CITACIÓNY REPRODUCCIÓN}

La revista Ciencia \& Desarrollo es una publicación semestral de carácter científico tecnológico, orientada a promover y difundir la investigación en campos multidisciplinarios. Su publicación es impresa y en idioma español.

Para la publicación en la revista Ciencia \& Desarrollo los artículos presentados deben ser inéditos y pueden ser: artículo científico original, artículo de revisión, carta al editor, reporte de caso clínico, artículo de opinión, reseña de líbro, ensayos, artículo breve. Los artículos publicados en esta revista pueden ser citados en otros documentos, siempre y cuando se indique la siguiente información: Revista Científica Ciencia \& Desarrollo. Tacna (Perú): Universidad Nacional Jorge Basadre Grohmann, número de la revista, página(s) y año de publicación. ISSN 2304-8891. Si la reproducción de artículos publicados en la revista, es con fines académicos y sin ánimo de lucro, puede realizarse si se incluye la información establecida en el párrafo anterior. Si su reproducción implica otros usos, debe solicitarse autorización escrita al Director de la Revista científica Ciencia \& Desarrollo.

La revista señala que la publicación de artículos no da derecho a remuneración alguna y que la responsabilidad del contenido de los artículos es de los autores, inclusive en lo relacionado con la propiedad intelectual de otros autores $\mathrm{y} / \mathrm{o}$ fuentes.

La revista científica Ciencia \& Desarrollo recibe artículos de las ciencias básicas y aplicadas, tecnológicas y de ingenieria.

\section{TIPOS DE ARTÍCULOS}

2.1. Artículo científico original: La extensión del desarrollo no debe ser más de 12 páginas o caras. La redacción es en tercera persona. Dado que la finalidad de un artículo científico es comunicar los resultados de investigaciones, ideas y debates de una manera clara, concisa y fidedigna, se tomará en cuenta los principios fundamentales de la redacción cientifica: precisión, claridad y brevedad. Además de los componentes del artículo cientíico deben cumplir con las siguientes características.

Su estructura es del siguiente tipo

Título: Debe contener en esencia el problema que está planteado, ser claro y preciso. Consta de 10 a 15 palabras, en caso de ser muy largo utilizar un subtitulo conciso. Su redacción es en mayúscula, respetando la redacción de las denominaciones de nombres científicos.

Title: Es el título traducido correctamente en idioma inglés.

Autor/es: Si el trabajo ha sido realizado en equipo, debe colocarse como primer autor, el que tuvo mayor responsabilidad en la realización del trabajo.

Afiliación. Institución a la cual pertenecen los autores (especificar departamento o área por cada autor)

Resumen: Debe contener en forma escueta los objetivos, planteamiento del problema, materiales y métodos, y resultados. El resumen y abstract se redactan en un promedio de 200 a 250 palabras. Se redacta en tiempo pasado. Debe ser escrito en un solo párrafo, separando las oraciones con punto seguido Sólo las palabras clave deben ir como punto aparte.

Palabras clave. No deben ser menores a dos ni mayores a cinco, ordenadas alfabéticamente.

Abstract. Es el resumen con una correcta traducción al inglés.

Key Word. Palabras clave correctamente traducidas al inglés.

Introducción: El autor debe procurar que todo el trabajo forme una unidad desde el inicio hasta el final, coherente y relacionado. En esta parte se expone el problema, se informa lo que se conoce del mismo, se hará una revisión bibliográfica pertinente, se discute el objetivo general, fin principal y la hipótesis (si lo hubiera).

Material y Métodos: Descripción de los recursos humanos, materiales físicos, financieros y técnico administrativos empleados; explicación detallada del método empleado, señalando las técnicas estadísticas, si se utilizaron.

Resultados: Se expondrá en forma clara siguiendo una secuencia lógica. Se exponen mediante figuras o tablas, donde se exponen la información resumida que confirman o no la(s) hipótesis y objetivos específicos planteados en la investigación. Deben limitarse a describir los propios hallazgos encontrados, evitando adelantar interpretaciones o comparaciones. Se redacta en pasado.

Discusión: En esta parte el autor podrá comparar, discutir, argumentar sus resultados con cl de otros autores. Tomar en cuenta que los resultados se exponen y no se recapitulan. De esta discusión se originarán puntos de partida para nuevas investigaciones. Se redacta en presente.

Conclusiones: Deberán ser redactadas en forma clara y concisa.

Agradecimiento: Incluirlos solamente si los hubiera, y solo se menciona a quienes contribuyeron con un apoyo muy importante (técnico) o las instituciones que han financiado la investigación (si fuera el caso).

Referencias Bibliográficas: Deben aparecer solamente las referencias bibliográficas utilizadas por el autor en la realización de la investigación y que son mencionadas en la redacción del artículo científico. Serán presentadas en el orden correspondiente al estilo bibliográfico. Considerar que si en el artículo hay quince citas, también debe haber quince referencias bibliográficas.

2.2. Articulo de Revisión: Debe contener: Título en español e inglés; Autor(es); Resumen; Palabras Clave; Abstract; Key Word; Introducción, Método utilizado para localizar y seleccionar los artículos relevantes sobre el tema. Análisis y comparación de los resultados encontrados, coincidencias y discrepancias; conclusiones; recomendaciones; referencias bibliográficas.

2.3. Reporte de Caso Clínico: Debe contener: Titulo en español y en inglés, Autor(es), Resumen, Palabras clave, Abstract, Key Word, Introducción, Anamnesis, Examen clínico, Exámenes auxiliares (laboratorio y gabinete), Etiologia, Diagnóstico preliminar (presuntivo), Tratamiento, Evolución y complicaciones (si las hubiera), Diagnóstico definitivo, Histopatología (si las tuviera), Discusión, Referencias Bibliográficas, Fotografias antes y después del tratamiento. En las fotos del rostro de una persona debe cubrirse los ojos para preservar su identidad, para mostrat el rostro completo se debe contar con el consentimiento escrito, firmado por el paciente y con impresión dactilar de su dedo índice.

2.4. Artículo de Opinión: Debe contener: Título en español y en inglés; nombre y apellidos del Autor(es), Introducción, Conclusiones, Referencias Bibliográficas.

2.5. Reseña de Libro: De contenido abierto en cuanto al contenido de la reseña. Sin embargo, será obligatorio considerar: Título del libro en mayúsculas, el nombre del autor o autores (en minúsculas, excepto las primeras letras del nombre y apellido), la edición, la editorial, el año de publicación, el ISBN (si tuviera) y el número de páginas. Así mismo, la carátula del libro, la editorial (escaneado a colores). El contenido no debe exceder de dos páginas. Al final de la reseña se debe colocar el nombre del autor de la reseña, indicando además, la licenciatura y los grados académicos (si los tuviera), así como los principales cargos académicos o institucionales que desempeña.

2.6. Ensayo Científico y Artículo Informativo: La estructura es: Título en español o inglés; nombre y apellidos del Autor(es); Resumen y Palabras Clave; 
Abstract y Key Words; Introducción; Desarrollo Temático; Conclusiones; Referencias Bibliográficas.

2.7. Carta al Editor: Debe contener: Título en español y en inglés, Autor y filiación institucional, Inicio mencionando la razón objetivo de la carta; si fuera necesario, sólo una tabla o una figura; Razón del planteamiento de la opinión, Discusión de resultados y/o recomendaciones, Referencias Bibliográficas, no más de seis referencias bibliográficas. Correo electrónico.

\section{ESTILO DE PRESENTACIÓN}

Los artículos a ser publicados, deberán cumplir los requisitos que a continuación se detalla:

DE LA PRESENTACIÓN: Se presentará 01 ejemplar impreso de un aproximado de 12 páginas y un CD. Los artículos deber ser preparados en formato MS Word utilizando la fuente Times New Romas de tamaño 10, a espacio sencillo. La primera página del artículo debe incluir; El Título (español e inglés), nombre de autor (es), afiliaciones, abstract y palabras clave. Y utilizarán la misma fuente con distinto tamaño y estilo.

DEL TAMAÑO Y MÁRGENES: En tamaño A4 $(210 \times 297 \mathrm{~cm})$. Los márgenes deben ser configurados de la siguiente manera: superior e izquierdo: 2.5 $\mathrm{cm}$; inferior $y$ derecho: $2 \mathrm{~cm}$. No se permite agregar pie de página ni encabezados. Las referencias y la bibliografia se agregarán al finalizar el texto.

DEL TÍTULO: El título será escrito en español y en inglés, en tamaño 16 y 14 respectivamente; nombre(s) de autor(es) en tamaño 12 ; la afiliación o institución/área a la que pertenece, dirección y correo electrónico (tamaño 12). El titulo y autor(es) van en negrita y deben estar centrados.

DEL RESUMEN: Resumen (Abstract) del artículo y palabras clave, español y en inglés. Cada artículo debe incluir un resumen de no más de 200 palabras en la primera página, seguido por una lista de palabras clave. El resumen debe ser conciso y las palabras clave deben estar justificadas de ambos lados (izquierdo y derecho)

DEL TEXTO: Estará escrito en dos columnas.

DE LAS REFERENCIAS Y CITAS: Utilizar el estilo de referencias bibliográficas acorde a su investigación. El formato de las citas y referencias bibliográficas que se utilizará será: en las ciencias de la salud (con excepción de Psicología) la norma Vancouver; y en las ciencias sociales, ciencias humanas, ciencias naturales y Psicología, la norma APA.

DE LAS SECCIONES: El título de una sección debe estar en fuente Times New Roman, tamaño 10 y en negrita, escrito con letras mayúsculas. Debe estar alineado a la izquierda a partir de la introducción, excepto las referencias bibliográficas.

DE LAS SUBDIVISIONES: El título de las subdivisiones debe estar alineado a la izquierda. La fuente a utilizar es Times New Romas tamaño 10 y en negrita y sólo las letras iniciales de cada palabra serán escritas en mayúscula.

DE LAS FIGURAS O TABLAS: Podrá incluirse fotografias, gráficos, tablas o imágenes, etiquetándolos únicamente como figuras o tablas según convenga.

Las figuras serán identificadas con la etiqueta Figura $\mathbf{N}^{\circ}$, numeradas con números arábigos de manera consecutiva, la cual será ubicada al pie de la figura. Las figuras, deberán adjuntarse adicionalmente como archivo de imagen (jpg o png).

Las tablas serán identificadas con la etiqueta Tabla $\mathbf{N}^{\circ}$, numeradas con números arábigos de manera consecutiva y ubicada en la cabecera de la tabla de forma centrada.

Las figuras y tablas a utilizar deberán ser insertadas en el punto apropiado del texto, debe ser mencionada en el texto al menos una vez y antes de su aparición.

DEL IDIOMA, ESTILO Y CONTENIDO: Los artículos deben presentarse en español. El título y el resumen están inscritos en español e inglés. La ortografia y puntuación deben escribirse con estilo sencillo y directo. Utilice estructuras simples para las oraciones, así como vocabulario común y básico Defina o explique el vocabulario técnico con sencillez. Explique acrónimos cuando aparezcan en el texto por primera vez. La presentación de unidades de medida y valores numéricos se realizará conforme al Sistema Internacional de Unidades, asimismo, debe utilizarse la coma "," para la separación decimal.

IMPORTANTE: Los autores son responsables de asegurarse que su trabajo sea conducido de una manera responsable yética. La estructura y estilo son tan importantes como el contenido. Antes de escribir su artículo, le recomendamos que lea la amplia literatura disponible para informarse respecto a cómo escribir un buen artículo técnico.

\section{PROCESO DE RECIBO, SELECCIÓN YEVALUACIÓN}

\subsection{Recepción de los artículos}

Las convocatorias para la recepción de artículos se cierran el 30 de abril y 30 de setiembre de cada año. Los artículos se entregan en formato impreso en la Oficina General de Investigación de la Universidad Nacional Jorge Basadre Grohmann sito en Ciudad Universitaria Av. Miraflores s/n, Tacna, Perú, o son enviados mediante correo electrónico a la cuenta coin@unjbg.edu.pe.

Junto al artículo (construido en un procesador de textos y guardado en formato .doc o docx) se envían las figuras presentadas en él, debidamente identificadas (en formato jpg o png con alta calidad), los datos de los autores debidamente diligenciados.

El formato impreso debe enviarse mediante carta de presentación acompañada del original impreso en papel, más un CD en formato de procesador de textos.

4.2. Proceso de selección

Para que un artículo sea admitido en la revista Ciencia \& Desarrollo debe cumplir con los parámetros de forma establecidos por la revista.

Los autores que decidan retirar sus artículos del proceso de selección, no implica que pierdan la posibilidad de presentarlo para ediciones futuras.

En los artículos que a pesar de ser aceptados, se detecte algún tipo de plagio no serán admitidos para la impresión final.

Después de un tiempo prudencial, el director del comité editorial comunicará al autor sobre la aceptación de su artículo. 4.3. Proceso de Evaluación

La primera evaluación la realiza el Comité Editorial, sobre el cumplimiento de los requisitos formales y la adecuación al interés temático de la Revista, de la cual se envía un mensaje a los autores donde se señala su resultado. En caso de corresponder al interés de la revista, se indican a los autores que aquellos aspectos a ajustar y la fecha limite para hacer la nueva entrega.

Comprobado el cumplimiento de los requisitos formales, el artículo es enviado a evaluación por parte de árbitros expertos, integrantes del Comité Científico de la revista. Este arbitraje se hace de forma confidencial (doble ciego; los árbitros desconocen los nombres de los autores y viceversa), mediante el diligenciamiento de un formato específico, que solicita su concepto sobre la conveniencia o no de su publicación, y las recomendaciones para mejoramiento del artículo. El resultado de la evaluación se informa a los autores oportunamente mediante correo electrónico, y en caso de requerirse, se indica la fecha para la entrega de los documentos ajustados, con el fin de que los árbitros emitan su concepto definitivo sobre la condición de publicable o no. 